\title{
Le travail des enfants : uniquement un problème de pauvreté?
}

Effet de la situation économique des ménages sur le travail des enfants au Cameroun en 2007

Child labour: an entierly poverty related problem? The effects of household economic situiation on child labour in Cameroon in 2007

\section{Cosmas Bernard Meka'a et Olivier Ewondo Mbebi}

\section{(2) OpenEdition}

Journals

Édition électronique

URL : http://journals.openedition.org/travailemploi/6683

DOI : 10.4000/travailemploi.6683

ISSN : $1775-416 \mathrm{X}$

Éditeur

DARES - Ministère du Travail

Édition imprimée

Date de publication : 1 juillet 2015

Pagination : 5-19

ISSN : 0224-4365

Référence électronique

Cosmas Bernard Meka'a et Olivier Ewondo Mbebi, « Le travail des enfants : uniquement un problème de pauvreté ? », Travail et Emploi [En ligne], 143 | juillet-septembre 2015, mis en ligne le 01 juillet 2017, consulté le 10 décembre 2020. URL : http://journals.openedition.org/travailemploi/6683 ; DOI : https:// doi.org/10.4000/travailemploi.6683 


\title{
Le travail des enfants: uniquement un problème de pauvreté ? (*) Effet de la situation économique des ménages sur le travail des enfants au Cameroun en 2007
}

\author{
Cosmas Bernard Meka'a ${ }^{(* *)}$, Olivier Ewondo Mbebi ${ }^{(* *)}$
}

\begin{abstract}
Cet article analyse les déterminants du travail des enfants âgés de 5 à 14 ans au Cameroun, en insistant sur les facteurs de vulnérabilité (état de pauvreté et chocs négatifs sur les revenus). L'étude utilise les données microéconomiques de la troisième Enquête camerounaise auprès des ménages (Ecam III). Le modèle probit bivarié mobilisé montre que le niveau de vie mesuré par le revenu a un effet négatif sur le travail des enfants et positif sur leur scolarisation. Ces effets sont néanmoins faibles après instrumentation du revenu. Par ailleurs, les variables de choc indiquent qu'une baisse brutale de revenu est positivement associée à un travail plus fréquent des enfants, tandis que la possession de terres, dont l'exploitation nécessite de la force de travail, tend à l'accroître.
\end{abstract}

Dans le rapport issu de la conférence internationale du travail qui s'est tenue à Genève en 2002, le Bureau international du travail (BIT) plaidait pour «un avenir sans travail des enfants» et pour que l'abolition effective de ce phénomène soit l'«un des impératifs les plus urgents de notre époque» (BIT, 2002). Le travail précoce des enfants pourrait en effet compromettre leur développement physique et mental et, au plan national, réduire les capacités d'accumulation de capital humain (ANKER, 2000; Ravallion, Wodon, 1999). La lutte pour le faire disparaître mobilise par conséquent des organismes internationaux ${ }^{(1)}$, des organisations non gouvernementales ainsi que les pouvoirs publics nationaux ${ }^{(2)}$.

En dépit de ces efforts répétés, le travail des enfants persiste. Les estimations récentes soulignent que près de 121 millions d'enfants âgés de 5 à 14 ans (soit un taux de 9,9\%) travaillent actuellement dans le monde (Diallo et al., 2013). La situation de l'Afrique au sud du Sahara est particulièrement

\footnotetext{
(*) Nous remercions vivement les rapporteurs anonymes de la revue pour leurs remarques et suggestions.

(**) Groupe d'études et de recherches en économie appliquée (Gerea); Groupe de recherche en économie et gestion (Greg); Faculté des sciences économiques et de gestion appliquée (Fsega), université de Douala, Cameroun; bmekaa@yahoo.fr

$(* * *)$ Greg; Faculté de génie industriel (FGI), université de Douala; oliewondo1@yahoo.fr

(1) Tels que l'Organisation internationale du travail (OIT), le Fonds des Nations unies pour l'enfance (Unicef) et la Banque mondiale.

(2) Aux yeux de l'opinion publique occidentale, chaque pays devrait se doter de lois interdisant le travail des enfants ou instaurant une obligation scolaire. Lorsqu'on estime qu'un pays n'œuvre pas assez en ce sens, une interdiction extérieure est par ailleurs jugée acceptable (DUMAS, LAMBERT, 2008).
}

préoccupante car, selon la même source, $21,7 \%$ d'enfants de cette tranche d'âge y travaillent.

L'analyse du travail des enfants est cependant une réalité complexe. Les mesures visant à lutter contre ce phénomène peuvent aboutir à des effets pervers. En effet, l'impact sur le niveau de vie des enfants et de leurs familles pourrait se révéler négatif s'ils étaient ainsi privés d'une source de revenu indispensable à leur survie. Dans les pays en développement, de très nombreux ménages - notamment des campagnes - sont pauvres et sujets à des chocs négatifs, les mécanismes formels permettant de les compenser (accès aux marchés financiers, existence d'assurances) étant par ailleurs limités, et souvent même inexistants. Les ménages ont alors tendance à recourir au travail des enfants comme instrument de réponse (BeEgle et al., 2006; Guarcello et al., 2010). L'incertitude induite par ces chocs affecte de ce fait l'investissement en capital humain des enfants en âge de scolarisation, les ménages effectuant un arbitrage entre école et travail afin d'absorber les impacts des chocs négatifs.

Comme nous allons le détailler plus loin, la littérature avance plusieurs arguments afin d'expliquer le travail des enfants. Depuis l'article de Kaushik BAsU et Pham Hoang VAN (1998), le facteur le plus couramment avancé est la pauvreté des ménages. On considère en effet que les ménages ne prennent la décision de faire travailler les enfants que si le revenu familial hors contribution des enfants descend en dessous du seuil de subsistance ${ }^{(3)}$. Cependant, la relation entre pauvreté et

(3) Ces auteurs qualifient cette hypothèse d' «axiome luxueux » de pauvreté, $c f$. infra. 
travail des enfants a été remise en question par un certain nombre de travaux empiriques ${ }^{(4)}$ : certains trouvent à l'inverse que la richesse des ménages, quand elle est mesurée par leur dotation en terres, est une des causes principales du travail des enfants (Bhalotra, Heady, 2003). À la suite de ces auteurs, d'autres facteurs de vulnérabilités du ménage, tels que le chômage des parents, le décès du père et/ ou de la mère, ou la baisse du revenu du ménage, ont été avancés comme des explications possibles du travail des enfants. Leur prise en compte permet d'apporter un éclairage sur les politiques à adopter pour réduire le phénomène.

L'objectif de cet article est précisément de mettre en évidence les déterminants du travail des enfants en utilisant les données de la troisième Enquête camerounaise auprès des ménages (Ecam III), qui a été réalisée en 2007 par l'Institut national de la statistique (INS). Nous examinons l'influence de différents facteurs de vulnérabilité des ménages, et non seulement la pauvreté monétaire. Aucune analyse de ce type n'a, à notre connaissance, été réalisée s'agissant du Cameroun.

D'après les données de l'Ecam III, 28,8\% des enfants âgés de 5 à 14 ans y travaillent. L'incidence est de surcroît largement plus élevée en milieu rural qu'en milieu urbain (40,7\% contre 16,6\%). Dans le même temps, le taux net de scolarisation dans le primaire est estimé à $79,8 \%$ en 2007 ; encore loin de la scolarisation universelle, il est resté inchangé depuis 2001. Dans l'ensemble, la structure démographique du pays est jeune : plus de deux Camerounais sur cinq $(41 \%)$ sont âgés de moins de 15 ans. Par conséquent, le ratio de dépendance(5) s'élève à $80,1 \%$. Par ailleurs, le pays a connu, dans la seconde moitié des années 1980, une crise économique dont les conséquences sur le niveau de vie des populations sont toujours perceptibles ${ }^{(6)}$. D'après l'INS (2008), la pauvreté est ainsi importante : sur une population d'environ 17,9 millions d'habitants en 2007, 39,9\% vivent en dessous du seuil de pauvreté, soit à peu près la même proportion qu'en 2001 (40,2\%), en dépit des importantes réductions de dettes obtenues durant cette période ${ }^{(7)}$; les populations rurales sont parti-

(4) Voir par exemple, Canagarajah, Coulombe (1997), Nielsen (1998), Maitra, Ray (2002). Kaushik Basu et Zafiris Tzannatos (2003) citent d'ailleurs quelques autres recherches critiquant l'hypothèse de pauvreté.

(5) Le ratio de dépendance représente le rapport entre les dépendants - la population âgée de moins de 15 ans ou de plus de 64 ans - et la population en âge de travailler - les personnes âgées de 15 à 64 ans.

(6) La crise financière internationale survenue en 2007 et 2008 n'a pas arrangé la situation car elle a eu pour effet la chute brutale de la demande et donc du prix des matières premières produites au Cameroun et à visée d'exportation, telles que le pétrole, le bois, le caoutchouc, le coton et l'aluminium (UCW, 2012).

(7) En effet, le Cameroun est sorti à cette période du programme «Initiative pays pauvres très endettés », ce qui a eu pour conséquence de relâcher les contraintes liées au service de la dette. culièrement affectées, puisqu'elles concentrent $87 \%$ des ménages en situation de pauvreté au Cameroun. Les indicateurs de pauvreté non monétaire pointent également une situation préoccupante. D'après le rapport de l'UCW (Understanding children's work), Cameroun, comprendre le travail des enfants et l'emploi des jeunes $(2012$, p. 8), «plus de la moitié de la population n'a pas accès aux installations sanitaires améliorées, plus d'un quart n'a pas accès à une source d'eau améliorée et trois cinquièmes à l'électricité». En outre, l'occurrence d'événements ayant des conséquences négatives sur les revenus ${ }^{(8)}$ conjugués à des marchés du travail, de la terre ou du crédit déficients augmente le coût d'opportunité du temps des enfants, ce qui est susceptible d'accroître leur participation au travail et, de façon concomitante, de réduire leur temps de scolarisation.

Après avoir synthétisé la littérature traitant des déterminants du travail des enfants, nous décrivons le modèle économétrique adopté dans l'article, puis précisons les données et variables utilisées dans l'analyse. Nous présentons ensuite des statistiques descriptives ainsi que les principaux résultats des estimations réalisées.

\section{Les déterminants du travail des enfants : une revue de la littérature}

Depuis une dizaine d'années, la question des déterminants du travail des enfants fait l'objet d'une attention particulière de la part des économistes du développement. Mais c'est sous l'impulsion de BASU et VAN (1998) que la théorie s'est développée au cours de la période récente. Leur analyse porte essentiellement sur les conditions de vie des ménages et l'interaction entre le travail des enfants et celui des adultes. Elle s'appuie sur un modèle statique de microéconomie des ménages dont l'hypothèse principale, appelée "axiome de luxe», stipule qu'un ménage fait travailler ses enfants si son revenu, sans celui de ces derniers, descend en dessous du seuil de subsistance du ménage. Le travail des enfants y est lié à la pauvreté, elle-même assimilée à l'insuffisance du revenu parental.

Cet axiome a fait l'objet de nombreuses vérifications empiriques qui confirment l'impact de la pauvreté sur le travail des enfants. NielsHugo Blunch et Dorte Verner (2000), à partir d'un modèle probit simple, mettent en évidence une relation positive et significative entre la pauvreté du ménage et le travail des enfants au Ghana. Jean-Pierre LACHAUd (2008), quant à lui, utilise un modèle probit bivarié et démontre que la pauvreté monétaire des ménages, mesurée par les dépenses

(8) En 2007, on dénombre plus de 450000 enfants (soit 11\%) ayant perdu au moins un parent (UCW, 2012). 
de consommation par tête, influence positivement le travail des enfants âgés de 5 à 14 ans au Burkina Faso. Il montre par ailleurs qu'il existe une corrélation négative entre travail et scolarisation des enfants. Eric V. EDMONDS (2005), dans son étude fondée sur des données de panel au Vietnam, suggère qu'une réduction sensible du travail des enfants est possible lorsque le statut économique du ménage s'améliore et pointe que, sur la période 1993-1998, près de $80 \%$ de la baisse du travail des enfants provient de l'amélioration des conditions économiques observées dans le pays.

Néanmoins, dans d'autres études, la corrélation entre pauvreté et travail des enfants est faible, voire absente. À partir de données péruviennes, Ranjan RAY (2000) conclut à l'absence de lien car les enfants peuvent combiner travail et école, quel que soit le statut du ménage. Il en est de même pour Peter Jensen et Helena S. Nielsen (1997) en Zambie, puis Sudharshan Canagarajah et Harold Coulombe (1999) au Ghana, qui ne trouvent pas non plus de relation significative entre le travail des enfants et la pauvreté des ménages. Pour ces auteurs, la pauvreté n'est pas le premier facteur expliquant ce phénomène.

Bien que leur cadre d'analyse permette de mieux comprendre les conditions de mise au travail des enfants, la principale critique formulée à l'égard du modèle de Basu et Van est qu'il ne tient pas compte d'autres facteurs - en dehors de la pauvreté des ménages - susceptibles d'influer sur l'offre du travail des enfants. Des travaux plus récents se sont servis des enquêtes sur les ménages réalisées dans plusieurs pays en développement pour tenter de mieux l'expliquer. Ces études ont pris diverses orientations. Certaines se sont intéressées à l'accès au crédit et aux imperfections du marché du travail et de la terre, tandis que d'autres ont analysé 1'effet de chocs négatifs dans la trajectoire des ménages.

L'accès au crédit par les ménages joue un rôle déterminant dans la participation des enfants au travail. En effet, lorsque le marché du crédit fonctionne, il devient possible pour les parents d'emprunter afin de financer la scolarisation des enfants. Or, dans la plupart des pays en développement, ces marchés sont imparfaits. Dans ces conditions, la difficulté d'accès au crédit incite les parents à faire travailler les enfants.

Dans une étude portant sur l'Afrique du Sud, EDMONDS (2006) compare le statut d'enfants habitant avec un adulte éligible à une pension de retraite à celui d'enfants habitant avec des adultes qui ne peuvent en bénéficier. Le fait de recevoir une pension de retraite constitue un revenu futur certain, qui peut servir de garantie à un emprunt. L'auteur observe que les enfants résidant avec un adulte éligible vont en moyenne plus à l'école et travaillent moins. Ces résultats confirment que la possibilité d'emprunter sur des revenus futurs (ici la pension de retraite) permet aux ménages d'investir dans l'éducation de leurs enfants. D'autres auteurs ${ }^{(9)}$ obtiennent des résultats similaires et trouvent une relation significative entre travail des enfants, scolarisation et imperfection du marché du crédit dans divers pays. Ils soulignent qu'en présence de contraintes de crédits, le travail des enfants peut devenir un recours toutes les fois que les parents sont dans l'impossibilité de faire un arbitrage intertemporel concernant leurs ressources. Ces différentes études montrent que le travail des enfants est utilisé par les parents comme un substitut de l'emprunt avec, pour conséquence, un transfert des revenus du futur vers le présent. Il constitue ainsi non seulement une forme d'auto-assurance, mais aussi un filet de sécurité que les ménages pauvres utilisent pour se prémunir contre les imperfections du marché du crédit.

Un autre facteur explicatif souvent considéré dans la littérature concerne les imperfections du marché du travail et de la terre. Ainsi, confronté à ces imperfections, un ménage possédant de vastes terres en zone rurale peut préférer employer ses enfants plutôt que d'investir dans l'éducation de ces derniers. En utilisant les données pakistanaises et ghanéennes pour analyser le "paradoxe de richesse ${ }^{(10)}$ », Sonia Bhalotra et Christopher HEADY (2003) établissent que les ménages fortement dotés en terres tendent à faire travailler leurs enfants tant qu'ils ne peuvent pas compter sur une main-d'œuvre extérieure et qu'ils éprouvent des difficultés à mettre leurs terres en location. La terre étant une importante source de richesse en milieu rural, ces auteurs trouvent que les enfants des ménages ruraux riches en terres sont en moyenne moins scolarisés et plus actifs que ceux des ménages dénués de terres. Christelle Dumas (2007), dans son étude portant sur le Burkina Faso, montre que la possession de terres accroît le travail des enfants en cas de problèmes d'aléa moral concernant les travailleurs adultes sur le marché du travail(11). Elle met en évidence que, pour toutes les catégories de ménage, il existe une relation en $\mathrm{U}$ entre le temps de travail des enfants et la quantité de terres cultivables (12).

Les études récentes de Delphine Boutin (2012) et Christelle Dumas (2013) établissent une relation

(9) Tels que par exemple : Jensen, Nielsen (1997); Baland, Robinson (2000); Dehejia, Gatti (2002); Guarcello et al., (2010).

(10) L'expression est utilisée pour décrire la situation de ménages «riches» faisant travailler leurs enfants.

(11) Certains travailleurs adultes prennent le risque de diminuer ou de dégrader leur offre de travail, car ils se placent dans la perspective d'employer leurs enfants au sein des exploitations agricoles familiales.

(12) Autrement dit, le travail des enfants diminue lorsque les ménages possèdent des quantités relativement faibles de terres cultivables, mais augmente à mesure que ces quantités sont de plus en plus élevées. 
positive entre le travail des enfants et certaines imperfections de marché, respectivement au Mali et à Madagascar. Boutin (2012) analyse le «paradoxe de richesse» en testant la relation entre la possession de terres et le travail domestique des enfants. À partir d'un modèle logit, elle trouve que la demande en main-d'œuvre infantile au sein de la famille augmente avec la propriété foncière. En outre, plus la surface cultivable est grande, moins les enfants ont tendance à travailler à l'extérieur de l'exploitation familiale. Dumas (2013) arrive à la conclusion selon laquelle la possession de terres s'accompagne d'une diminution du travail des enfants lorsque les imperfections du marché du travail diminuent. Par conséquent, l'une des mesures possibles de lutte contre le travail des enfants consisterait à favoriser la concurrence sur le marché du travail.

Les chocs que subissent les ménages constituent également des facteurs de vulnérabilité qui incitent à faire travailler les enfants. En effet, en l'absence de mécanismes de prévoyance ou de réparation (accès aux marchés du crédit et de l'assurance, système de sécurité sociale, etc.), les ménages qui sont confrontés aux chocs transitoires de revenu ou aux chocs non monétaires ont tendance à retirer leurs enfants de l'école afin de les faire travailler et de lisser ainsi leur consommation. Kathleen BEEGLE et ses coauteurs (2006) cherchent à évaluer dans quelle mesure les chocs transitoires sur les revenus des ménages conduisent à un accroissement du travail des enfants, et si l'accès au crédit diminue ces effets. En utilisant les données d'un échantillon de huit ménages en Tanzanie, les auteurs parviennent à montrer que les chocs agricoles ${ }^{(13)}$ entraînent une augmentation substantielle du travail des enfants. Par ailleurs, les ménages possédant des actifs peuvent les utiliser non seulement pour faire face aux chocs, mais aussi comme garantie d'accès au crédit. Alain DE JANVRY et ses coauteurs (2006), à partir des données issues de l'expérience Progresa ${ }^{(14)}$ au Mexique, pointent que des chocs idiosyncratiques (chômage ou maladie du chef de famille) ou covariants (désastres naturels) poussent les parents à adopter des stratégies de gestion du risque : ils retirent leurs enfants de l'école pour les mettre au travail. Par la méthodologie d'appariement en score de propension (propensity score matching [PSM]) sur des données du Guatemala, Lorenzo GuARCELLO et ses coauteurs (2010) cherchent à savoir dans quelle mesure les facteurs de risque et de vulnérabilité tels que le rationnement de crédit et les chocs individuels ou collectifs influencent les décisions des ménages relatives à l'éducation et à l'offre de travail des enfants. Ils concluent que la

(13) Par exemple, les pertes accidentelles de récolte ou les catastrophes naturelles (induisant une baisse de revenu).

(14) Progresa est un programme de transfert de fonds conditionnel à une scolarisation et un suivi de santé régulier des enfants. survenance de ces chocs réduit le niveau d'éducation des enfants et augmente leur offre de travail. Dans le même ordre d'idées, dans une étude réalisée en Tanzanie, Amarakoon BANDARA et ses coauteurs (2015) utilisent un modèle dans lequel les ménages maximisent leur utilité en fonction de leur consommation et du niveau d'éducation des enfants pour analyser l'impact des chocs monétaires (chocs agricoles) et non monétaires (décès des parents) sur le travail des enfants. Ils cherchent notamment à déterminer si l'accès au crédit et les actifs du ménage permettent de faire face à ces chocs. Il ressort de cette étude que les chocs agricoles ont un impact significatif sur le travail des enfants et que l'accès au crédit agit efficacement comme solution.

\section{Le modèle économétrique}

L'approche économétrique la plus simple pour analyser la probabilité qu'un enfant travaille est d'estimer une équation d'offre de travail grâce à un modèle logit ou à un modèle probit univarié. Ces modèles présentent cependant l'inconvénient de ne pas prendre en compte l'interaction entre les décisions de scolarisation et de travail des enfants. Il paraît donc préférable de recourir à des modèles multivariés qui permettent d'estimer à la fois une équation de participation au marché du travail et une équation de scolarisation. Trois méthodes ont été mises en œuvre dans la littérature sur l'offre de travail des enfants. Elles reposent sur des hypothèses différentes relatives à la manière dont les parents prennent la décision, ou non, de faire travailler leurs enfants. L'utilisation du modèle probit séquentiel implique que la décision d'allocation du temps de l'enfant est prise dans un ordre a priori déterminé, qui ne semble pas faire consensus ${ }^{(15)}$. Par ailleurs, l'hypothèse de l'indépendance des alternatives non pertinentes (independence of irrelevant alternatives [IIA]) qui est faite dans le modèle multinomial n'est vraisemblablement pas vérifiée dans le cas de la décision d'offre de travail et de scolarisation des enfants ${ }^{(16)}$. La meilleure solution paramétrique

(15) Ce modèle a en particulier été utilisé dans les études coordonnées par Christiaan Grootaert et Harry A. Patrinos (1998). La hiérarchie des options qui y a été choisie (et dont dépend le résultat) - l'école, le travail salarié, le travail au sein de l'entreprise familiale, l'inactivité (la préférence du parent allant décroissant) - est tout à fait contestable. GROOTAERT explique que les critères pris en compte pour effectuer ce classement sont, d'abord le bien-être de l'enfant, et ensuite, sa contribution économique. Mais, d'une part, les parents peuvent considérer que le bien-être de l'enfant sera supérieur s'il gagne de l'argent que s'il va à l'école, et, d'autre part, les parents préfèrent souvent que leur enfant travaille avec eux que pour un employeur qui peut le «maltraiter».

(16) Cette hypothèse suppose que les pourcentages de chances de faire tel ou tel choix sont indépendants les uns des autres. Il est probable que dans la réalité, le choix des ménages se fasse en considérant simultanément les avantages et inconvénients en termes d'utilité de toutes les options se présentant à eux. 
nous paraît donc être le modèle probit bivarié, qui suppose que le travail et la scolarisation des enfants sont des choix interdépendants.

De ce fait, nous supposons que l'école a pour effet de soustraire les enfants à de nombreuses activités. En utilisant un probit bivarié, on teste la probabilité pour les enfants de travailler et/ou d'aller à l'école. Ce type de modèle tient compte de l'existence de possibles distributions corrélées entre les deux équations de choix. Ainsi, dans ce modèle, il y a deux variables dépendantes binaires, l'une indiquant si l'enfant travaille, l'autre traduisant le fait d'aller à l'école. Formellement, la procédure du maximum de vraisemblance conditionnel en deux étapes ( $t w o-$ stage conditional maximum likelihood [2SCML]) décrite par Douglas Rivers et Quang H. VuONG (1988) a été utilisée afin d'obtenir des estimateurs non biaisés et d'estimer simultanément une équation de participation au travail (T) et une équation de scolarisation (E). Les équations réduites de ce modèle peuvent être exprimées comme suit :

$\left\{\begin{array}{l}T^{*}=\alpha_{1} R+\beta_{1} H+\gamma_{1} X+u \text { avec } T=1 \text { si } T^{*}>0,0 \text { sinon } \\ E^{*}=\alpha_{2} R+\beta_{2} H+\gamma_{2} X+v \text { avec } E=1 \text { si } E^{*}>0,0 \text { sinon }\end{array}\right.$

où $T^{*}$ et $E^{*}$ désignent des variables latentes non observables, reliées à l'utilité des parents concernant le fait de faire travailler leurs enfants et de les scolariser, $R$ le revenu du ménage ${ }^{(17),} H$ le vecteur des variables de chocs et $X$ le vecteur des variables de contrôle; $u$ et $v$ sont les termes aléatoires de moyenne nulle et de variance unitaire.

Cette méthode permet par ailleurs de corriger de l'endogénéité du revenu du ménage. De fait, l'intégrer directement dans les équations (1) et (2) peut être source de biais : le revenu doit être instrumenté car il est endogène à l'offre de travail des enfants ${ }^{(18)}$. Même si l'on observait le revenu de l'enfant, le soustraire au revenu familial ne résoudrait pas ce problème d'endogénéité puisque l'offre de travail des différents membres du ménage dépend vraisemblablement du fait que les enfants travaillent ou

(17) Plusieurs analystes estiment que la consommation est un meilleur indicateur de la pauvreté que le revenu. Mais, dans cette étude, nous utilisons le revenu suivant le raisonnement d'Aline Coudouel et de ses coauteurs (2002), pour lesquels la mesure de la pauvreté au moyen du revenu permet de faire la distinction entre les différentes sources de revenus. Lorsque de telles distinctions sont possibles, le revenu peut de fait être plus aisément comparé aux données en provenance d'autres sources, telles que les salaires, ce qui permet de procéder à une vérification de la qualité des données de l'enquête auprès des ménages. (18) Toutes choses égales par ailleurs, plus l'enfant travaille, plus le revenu du ménage est élevé. pas ${ }^{(19)}$. La première étape de notre procédure d'estimation consiste donc à régresser par les moindres carrés ordinaires le revenu $R$ sur les variables exogènes $Z$ (autrement dit, les variables de chocs et de contrôle, augmentées d'une ou plusieurs variables instrumentales) :

$$
R=\delta Z+w
$$

L'instrumentation du revenu est délicate car les enquêtes utilisées ne contiennent aucune variable ayant a priori les propriétés d'une variable instrumentale, c'est-à-dire étant corrélée avec le revenu mais pas avec le résidu de l'équation d'offre de travail des enfants. Certains auteurs ${ }^{(20)}$ proposent comme première stratégie d'instrumentation la prise en compte du revenu retardé de la période. Dans ce cas, il faut disposer d'un panel de ménages enquêtés en $t$ et en $t-1$. Malheureusement les données d'Ecam III ne nous permettent pas de mettre en œuvre cette méthode. Une deuxième stratégie d'identification consiste à instrumenter le revenu avec des variables relatives aux biens durables détenus par le ménage et aux caractéristiques du logement. Ces variables sont assez fortement corrélées avec le revenu mais souffrent de deux biais de sens contraire : un biais d'endogénéité (les biens durables peuvent avoir été acquis en partie grâce au revenu de l'enfant) et un «biais de développement» (les caractéristiques du logement, telle la présence d'eau courante, dépendent pour partie du niveau de développement de la zone de résidence et peuvent donc être corrélées avec la qualité de l'éducation). Une troisième stratégie d'instrumentation utilise un petit nombre de variables dont on peut s'attendre à ce qu'elles ne soient que faiblement corrélées avec les résidus de l'équation d'offre de travail des enfants. C'est le cas, par exemple, de l'âge du chef de ménage et du taux de salaire des adultes de la subdivision administrative (COURALET, 2003).

Nous utilisons cette dernière stratégie dans le cadre de notre étude. Plus précisément, nous considérons comme instrument du revenu du ménage le taux de salaire des adultes de l'arrondissement, ce qui constitue la première étape de notre méthode d'estimation. Dans la seconde étape, le modèle probit bivarié est estimé en incluant le résidu estimé de la première étape $(w)$ parmi les variables explicatives. D'où, si on fait l'hypothèse que $u$ et $w$ d'une part, et $v$ et $w$ d'autre part, ont des distributions normales jointes et que la distribution des termes

(19) Un certain nombre d'auteurs qui ont estimé des équations d'offre de travail des enfants ont pourtant utilisé cette stratégie. Elle est d'autant plus inadéquate que, pour la majorité des ménages, le revenu des enfants n'est pas connu puisqu'ils travaillent pour les membres du ménage et ne sont pas rémunérés (Couralet, 2003).

(20) Par exemple, Thomas (2000) 
d'erreur $\left(\varepsilon_{1}, \varepsilon_{2}\right)$ peut être approximée par une loi normale bivariée avec :

$$
\begin{gathered}
\mathrm{E}\left(\varepsilon_{1}\right)=\mathrm{E}\left(\varepsilon_{2}\right)=0 \\
\mathrm{~V}\left(\varepsilon_{1}\right)=\mathrm{V}\left(\varepsilon_{2}\right)=1 \\
\operatorname{Cov}\left(\varepsilon_{1}, \varepsilon_{2}\right)=\rho^{(21)}
\end{gathered}
$$

alors,

$$
\left\{\begin{array}{l}
T^{*}=\alpha_{1} R+\beta_{1} H+\gamma_{1} X+\lambda_{1} \mathrm{w}+\varepsilon_{1} \\
E^{*}=\alpha_{2} R+\beta_{2} H+\gamma_{2} X+\lambda_{2} \mathrm{w}+\varepsilon_{2}
\end{array}\right.
$$

\section{Données et définition des variables}

Comme indiqué plus haut, les données mobilisées dans cette étude proviennent de la troisième Enquête camerounaise auprès des ménages (Ecam III) réalisée en 2007. Conduite auprès d'un échantillon représentatif de 11391 ménages installés aussi bien en zone urbaine qu'en zone rurale, cette enquête fournit des informations détaillées sur tous les aspects de leurs conditions de vie, ce qui inclut leurs dépenses, revenus, patrimoine, accès au crédit, chocs monétaires et non monétaires et d'autres caractéristiques encore.

Précisons qu'Ecam III n'a pas été spécialement conçue pour l'étude du travail des enfants. Cependant, étant donné que l'objectif principal de l'enquête était d'actualiser le profil de pauvreté et les indicateurs des conditions de vie des ménages, elle comprend une rubrique sur le travail des enfants. $\mathrm{La}$ principale limite de l'enquête est qu'elle est constituée de données transversales correspondant à une seule année, alors que des données de panel auraient permis de mieux éliminer les biais éventuels liés aux variables inobservables. Un échantillon global des enfants de 5 à 14 ans a été constitué, ainsi que deux sous-échantillons en fonction de la zone de résidence : il contient 13716 enfants de la tranche d'âge considérée, dont 5122 habitent en zone rurale et 8594 en zone urbaine.

Dans l'article, le travail des enfants fait référence aux activités économiques mais aussi au travail domestique, pour au moins une heure au cours de la semaine de référence ${ }^{(22)}$. Par ailleurs, dans la mesure où la modélisation des déterminants du travail des enfants fait référence à leur scolarisation, nous appréhendons cette dernière, pour les individus de la même tranche d'âge, par la question : «Est-ce que l'individu fréquente actuellement l'école ?»

(21) Ce coefficient mesure la corrélation entre les termes aléatoires des équations et les facteurs omis.

(22) La question posée dans le questionnaire d'Ecam III est : «Au cours des sept derniers jours, la personne a-t-elle travaillé ne serait-ce qu'une heure, à son propre compte, comme employé rémunéré ou non, apprenti ou aide familial ?» Cette question couvre donc à la fois les activités économiques et domestiques.
Cette approche laisse ouverte la possibilité de choix interdépendants entre travail et scolarisation. Trois raisons justifient le choix de la tranche d'âge retenue. Premièrement, elle correspond dans la plupart des pays à celle de la scolarisation obligatoire (Couralet, 2003). Deuxièmement, le Cameroun a signé la convention 138 de l'OIT, qui stipule que l'âge minimum d'accès à l'emploi ne doit pas être inférieur à l'âge auquel cesse la scolarisation obligatoire ${ }^{(23)}$. Troisièmement, la législation camerounaise prévoit que les enfants âgés de plus de 14 ans peuvent être embauchés comme employés ou apprentis dans les entreprises.

Outre le revenu (24), central dans notre analyse, et dans la lignée des travaux mentionnés dans notre revue de littérature, nous introduisons des variables identifiant des chocs monétaires et non monétaires, ainsi que l'accès au crédit, comme facteurs pouvant expliquer la mise au travail des enfants au cours des douze derniers mois (période de référence commune aux trois chocs): pour les chocs monétaires, nous avons défini une variable binaire qui prend la valeur 1 si le chef de ménage a cessé ses activités et/ou a connu une baisse brutale de son revenu et 0 sinon; pour les chocs non monétaires, la variable définie prend la valeur 1 si l'enfant a perdu au moins un de ses parents et 0 sinon ${ }^{(25)}$; quant à l'accès au crédit, la variable prend la valeur 1 si le ménage a bénéficié d'un crédit sur la période, 0 sinon.

Les variables explicatives sont à la fois économiques et sociodémographiques dans la mesure où l'environnement familial et communautaire peut jouer un rôle décisif sur le travail des enfants (Dumas, 2004). Compte tenu des données collectées dans Ecam III, ces variables peuvent être regroupées en trois grandes catégories : les caractéristiques du ménage, celles de l'enfant et celles liées à l'environnement familial. Les variables sociodémographiques concernent les caractéristiques de l'enfant telles que son âge, son sexe et son statut par rapport au chef de ménage. Le fait par exemple d'être un enfant du chef de ménage peut conduire à une allocation du temps différencié par rapport aux autres enfants du groupe. Plusieurs travaux (Grootaert, Patrinos, 1998; Kobiané, 2009) montrent que ces derniers sont généralement plus actifs que ceux du chef de ménage. Plusieurs facteurs relatifs au ménage sont

(23) Toutefois des régimes d'exception sont prévus selon la nature de l'emploi exercé.

(24) Ecam III nous permet d'identifier trois composantes du revenu du ménage: le revenu tiré de l'activité principale, le revenu tiré de l'activité secondaire, les transferts (privés et institutionnels)

(25) La question posée dans Ecam III pour prendre en compte les chocs monétaires est la suivante: "Comment a évolué le revenu du chef de ménage par rapport aux douze derniers mois ?» Et pour considérer les chocs non monétaires, la question suivante a été posée : "L'enfant a-t-il perdu un de ses parents au cours des douze derniers mois ?» 
pris en compte. Premièrement, le niveau d'instruction et le statut sur le marché du travail du chef de ménage sont susceptibles d'avoir une influence sur le travail ou non des enfants. Il en est de même du sexe du chef de ménage, car lorsque ce dernier est géré par une femme, le taux de dépendance tend à être plus élevé (Lachaud, 2008). Deuxièmement, la composition démographique du ménage, autrement dit la taille du ménage mesurée par le nombre de personnes vivant dans le ménage ainsi que le nombre d'enfants à charge dans le ménage, peut également jouer. En principe, la probabilité que les enfants travaillent croît avec le nombre d'enfants dans le ménage, tandis que l'inverse prévaut pour la scolarisation. Pour ce qui est des facteurs liés à l'environnement, la localisation géographique des ménages ou des individus s'avère particulièrement importante. Deux raisons expliquent sa prise en compte. D'une part, l'absence de contrôle par celle-ci peut induire un biais sur l'effet des caractéristiques non géographiques sur le travail et la scolarisation des enfants. D'autre part, dans un pays agricole comme le Cameroun, la localisation géographique (notamment la spécification des zones agro-écologiques (26) ) est l'une des caractéristiques importantes déterminant le niveau de vie des ménages. Notre modèle prend également en compte les principaux actifs du ménage (possession de terres et d'une habitation inoccupée) et la distance par rapport à l'école primaire la plus proche.

\section{Analyse statistique des déterminants du travail des enfants}

Les résultats statistiques du tableau 1 montrent que plusieurs facteurs influencent le travail et la scolarisation des enfants, parmi lesquels l'âge, le niveau de vie, le lieu de résidence, l'accès au crédit, la détention de terres et les chocs négatifs.

Le niveau de vie semble un déterminant important du travail des enfants. En effet, pour les filles comme pour les garçons, l'incidence du travail baisse au fur et à mesure que le niveau de vie du

(26) En effet, le Cameroun rural est composé de trois principales zones agro-écologiques : la Forêt, les Hauts Plateaux et la Savane. La Forêt (centre, est, sud) se situe dans les zones équatoriales. Elle est propice à la culture du cacao, du palmier à huile, de l'hévéa, etc. Les Hauts Plateaux se trouvent dans les zones maritimes (littoral et sud-ouest) et riches en terres volcaniques (ouest, nord-ouest), favorables à l'agriculture (café, cultures maraîchères, etc.). Dans la Savane (nord, région de l'Adamaoua, extrême-nord), l'élevage bovin et la culture du coton et du mil sont très répandus. Elle est toutefois considérée comme la zone la plus pauvre du pays. En dehors de ces trois zones rurales, les zones urbaines principales se situent à Douala, capitale économique, Yaoundé, capitale politique et autour des villes secondaires. ménage s'élève. Par exemple, $10,7 \%$ des enfants issus de ménages très pauvres travaillent sans aller à l'école, contre $4,5 \%$ de ceux issus de ménages intermédiaires et $2,2 \%$ de ménages très riches. La tendance est la même pour les enfants qui travaillent en restant scolarisés $(40,9 \% ; 27,1 \%$ et $11,1 \%$ respectivement). Par contre la proportion de ceux qui vont uniquement à l'école augmente avec le niveau de vie (27).

Lorsqu'on tient compte du lieu de résidence, le tableau 1 révèle que, de manière générale, la proportion d'enfants travailleurs est plus élevée en milieu rural qu'en milieu urbain. En effet, 10,1\% des enfants habitant en zone rurale travaillent sans aller à l'école, contre $2,5 \%$ de ceux habitant en zone urbaine. L'inverse prévaut pour ceux qui vont uniquement à l'école, les taux étant de 52,8\% en milieu rural et de $78,2 \%$ en milieu urbain. Une analyse s'intéressant aux différentes zones agroécologiques révèle que les enfants des ménages issus de la Savane sont largement plus actifs que les autres, indépendamment du sexe. En effet, 38,6\% des enfants de la Savane travaillent - dont $8,2 \%$ n'ont pour seule activité que le travail et $30,4 \%$ combinent travail et école.

Pour ce qui est de l'accès au crédit, l'incidence du travail des enfants est plus élevée chez les ménages contraints, que chez les ménages ayant accès au crédit $(29,3 \%$ contre $22,9 \%)$. Inversement, les enfants sont davantage scolarisés dans ces derniers que dans les ménages contraints $(87,7 \%$ contre $84,6 \%)^{(28)}$. On observe aussi cette tendance selon que les ménages disposent ou non d'habitations non occupées. Enfin, il ressort du tableau 1 que les ménages qui subissent des chocs négatifs de revenu ont tendance à faire davantage travailler leurs enfants. C'est aussi le cas quand les ménages possèdent des terres cultivables.

Ces résultats préliminaires confirment qu'une large palette de facteurs est liée au travail des enfants. Ils ne sont toutefois pas indépendants les uns des autres, ce que montrent les analyses économétriques que nous présentons dans la section suivante.

(27) Par ailleurs, même si les ménages riches sont plus avantagés concernant la scolarisation, plus de huit enfants sur dix vont à l'école (y compris en travaillant) dans les ménages pauvres. Cette situation est peut-être due à la suppression des droits d'inscription dans l'enseignement primaire décidée par le gouvernement en 1999-2000.

(28) Notons tout de même que les écarts ne sont pas très importants. 
Tableau 1 : Incidence du travail et de la scolarisation des enfants par sexe et selon l'âge, le niveau de vie du ménage, le lieu de résidence et la zone agro-écologique (\%)

\begin{tabular}{|c|c|c|c|c|c|c|c|c|c|c|c|c|}
\hline \multirow[t]{2}{*}{ Sexe } & \multicolumn{4}{|c|}{ Garçons } & \multicolumn{4}{|c|}{ Filles } & \multicolumn{4}{|c|}{ Ensemble } \\
\hline & $\begin{array}{c}\text { Travail } \\
\text { seul }\end{array}$ & $\begin{array}{l}\text { École } \\
\text { seule }\end{array}$ & $\begin{array}{l}\text { Travail } \\
\text { et école }\end{array}$ & $\begin{array}{c}\mathrm{Ni} \\
\text { travail ni } \\
\text { école }\end{array}$ & $\begin{array}{c}\text { Travail } \\
\text { seul }\end{array}$ & $\begin{array}{l}\text { École } \\
\text { seule }\end{array}$ & $\begin{array}{l}\text { Travail } \\
\text { et école }\end{array}$ & $\begin{array}{c}\mathrm{Ni} \\
\text { travail } \\
\text { ni école }\end{array}$ & $\begin{array}{c}\text { Travail } \\
\text { seul }\end{array}$ & $\begin{array}{l}\text { École } \\
\text { seule }\end{array}$ & $\begin{array}{l}\text { Travail } \\
\text { et école }\end{array}$ & $\begin{array}{c}\mathrm{Ni} \\
\text { travail ni } \\
\text { école }\end{array}$ \\
\hline \multicolumn{13}{|l|}{ Tranche d'âge } \\
\hline 5-9 ans & 3,5 & 78,7 & 12,6 & 5,0 & 3,3 & 78,4 & 12,7 & 5,4 & 3,9 & 78,4 & 12,7 & 5,2 \\
\hline $10-14$ ans & 6,3 & 59,9 & 30,5 & 3,2 & 8,1 & 57,8 & 29,2 & 4,9 & 7,6 & 58,4 & 29,4 & 4,5 \\
\hline \multicolumn{13}{|l|}{ Niveau de vie (1) } \\
\hline Très pauvres & 10,9 & 41,3 & 40,1 & 7,6 & 11,9 & 39,3 & 40,9 & 7,7 & 10,7 & 40,3 & 40,9 & 7,9 \\
\hline Pauvres & 8,8 & 51,7 & 32,2 & 7,5 & 5,8 & 53,1 & 34,5 & 7,4 & 6,8 & 52,1 & 34,1 & 7,1 \\
\hline Intermédiaires & 5,7 & 66,3 & 21,8 & 6,1 & 4,2 & 61,7 & 27,1 & 7,0 & 4,5 & 70,5 & 27,1 & 7,5 \\
\hline Riches & 4,8 & 70,9 & 18,8 & 5,3 & 3,5 & 71,6 & 17,7 & 7,7 & 4,2 & 70,7 & 18,2 & 6,7 \\
\hline Très riches & 1,9 & 85,2 & 10,6 & 2,2 & 1,5 & 81,1 & 11,5 & 6,2 & 2,2 & 83,1 & 11,1 & 4,2 \\
\hline \multicolumn{13}{|c|}{ Zones de résidence } \\
\hline Rurale & 9,2 & 53,1 & 30,6 & 6,9 & 10,1 & 52,3 & 30,8 & 6,7 & 10,1 & 52,8 & 30,5 & 6,7 \\
\hline Urbaine & 2,2 & 79,1 & 13,8 & 4,8 & 3,5 & 77,9 & 12,2 & 6,2 & 2,5 & 78,2 & 14,1 & 5,1 \\
\hline \multicolumn{13}{|c|}{ Zones agro-écologiques } \\
\hline Yaoundé & 3,5 & 89,3 & 5,1 & 2,1 & 3,7 & 88,1 & 5,6 & 2,5 & 3,6 & 89,1 & 5,2 & 2,3 \\
\hline Douala & 3,2 & 89,6 & 5,1 & 2,1 & 3,4 & 89,1 & 5,1 & 2,4 & 3,3 & 89,3 & 5,1 & 2,1 \\
\hline Autres villes & 3,5 & 71,4 & 20,2 & 5,7 & 3,2 & 71,2 & 19,1 & 5,5 & 3,9 & 71,1 & 20,0 & 5,1 \\
\hline Forêt & 4,2 & 61,3 & 29,4 & 4,9 & 4,5 & 61,1 & 29,1 & 5,5 & 4,3 & 61,2 & 29,3 & 5,1 \\
\hline Hauts Plateaux & 2,1 & 68,1 & 27,1 & 3,1 & 1,9 & 66,7 & 28,2 & 3,1 & 2,2 & 68,1 & 27,1 & 3,1 \\
\hline Savane & 8,7 & 56,8 & 30,3 & 5,1 & 8,8 & 55,1 & 30,3 & 5,7 & 8,2 & 55,9 & 30,4 & 5,4 \\
\hline \multicolumn{13}{|l|}{ Chocs négatifs } \\
\hline \multicolumn{13}{|c|}{ Diminution du revenu } \\
\hline Oui & 7,4 & 57,1 & 27,3 & 8,1 & 8,9 & 57,5 & 26,2 & 8,6 & 8,5 & 57,3 & 27,7 & 8,5 \\
\hline Non & 5,2 & 70,1 & 18,3 & 6,3 & 7,2 & 69,5 & 17,2 & 6,1 & 5,1 & 70,5 & 18,1 & 6,1 \\
\hline \multicolumn{13}{|l|}{ Décès des parents } \\
\hline Oui & 9,3 & 55,3 & 27,2 & 8,2 & 11,8 & 54,5 & 25,2 & 8,4 & 10,5 & 55,8 & 26,2 & 8,4 \\
\hline Non & 6,1 & 63,7 & 22,5 & 7,4 & 5,9 & 65,9 & 21,2 & 7,1 & 5,7 & 64,5 & 22,6 & 7,2 \\
\hline \multicolumn{13}{|l|}{ Actifs } \\
\hline \multicolumn{13}{|l|}{ Accès au crédit } \\
\hline Oui & 5,9 & 71,7 & 16,3 & 6,2 & 7,4 & 70,1 & 16,1 & 6,4 & 6,1 & 70,9 & 16,8 & 6,4 \\
\hline Non & 5,4 & 65,4 & 19,4 & 6,3 & 6,7 & 66,5 & 21,5 & 5,4 & 8,8 & 64,1 & 20,5 & 6,7 \\
\hline \multicolumn{13}{|c|}{ Possession de terres } \\
\hline Oui & 8,3 & 60,1 & 23,4 & 8,2 & 7,8 & 61,9 & 21,9 & 8,6 & 8,3 & 60,2 & 22,9 & 8,6 \\
\hline Non & 6,2 & 69,3 & 16,3 & 8,2 & 5,1 & 71,7 & 15,1 & 8,1 & 5,2 & 71,5 & 15,1 & 8,2 \\
\hline \multicolumn{13}{|l|}{ Maison inoccupée } \\
\hline Oui & 4,5 & 68,2 & 21,1 & 6,1 & 4,2 & 69,5 & 20,1 & 6,2 & 4,1 & 69,7 & 20,1 & 6,2 \\
\hline Non & 5,2 & 64,5 & 23,8 & 6,4 & 6,8 & 63,3 & 24,5 & 5,2 & 6,2 & 64,6 & 23,8 & 5,2 \\
\hline Ensemble & 4,4 & 65,9 & 24,4 & 5,2 & 5,1 & 66,2 & 23,7 & 4,8 & 4,7 & 66,1 & 24,1 & 5,1 \\
\hline Effectif & 304 & 4548 & 1682 & 365 & 343 & 4517 & 1621 & 336 & 647 & 9065 & 3303 & 701 \\
\hline
\end{tabular}

(1) Le niveau de vie du ménage est défini selon le quintile de revenu mensuel par tête : quintile $1<134200$ francs CFA (soit 204,60 euros 2007); 134200 francs CFA $\leq$ quintile $2<195595$ francs CFA (soit 298,20 euros 2007); 195595 francs CFA $\leq$ quintile $3<284850$ francs CFA (soi 434,30 euros 2007); 284850 francs CFA $\leq$ quintile $4<439050$ francs CFA (soit 669,30 euros 2007); quintile $5 \geq 439050$ francs CFA. Le taux fixe de conversion du franc CFA avec l'euro est de 655,95 francs CFA pour 1 euro.

Lecture : $12,7 \%$ des enfants âgés de 5 à 9 ans travaillent en plus d'aller à l'école.

Source : Calculs des auteurs à partir de la base de données d'Ecam III.

\section{Déterminants du travail et de la scolarisation des enfants : approche en termes de choix interdépendants}

À l'aide d'un modèle probit bivarié faisant suite à une instrumentation du revenu du ménage par les taux de salaires observés dans l'arrondissement (29), nous mettons en évidence l'ampleur de l'association statistique correspondant aux différents déterminants de la scolarisation et du travail des enfants ( $c f$. tableau 2 pour les estimations conduites sur l'échantillon total; $c f$. annexes, tableau A2 pour les estimations relatives aux sous-échantillons ruraux et urbains).

(29) À titre de comparaison, le tableau A1 en annexes propose les résultats des estimations concernant les revenus non instrumentés. 
Tableau 2 : Probit bivarié des déterminants du travail et de la scolarisation des enfants âgés de 5 à 14 ans, relatifs au revenu instrumenté

\begin{tabular}{|c|c|c|c|c|c|c|}
\hline \multirow{2}{*}{ Variables } & \multicolumn{3}{|c|}{ Scolarisation } & \multicolumn{3}{|c|}{ Travail des enfants } \\
\hline & Coefficient & Écart-type & Effet marginal & Coefficient & Écart-type & Effet marginal \\
\hline Constante & $-3,10 * * *$ & 0,47 & $0,00 * * *$ & $2,72 * * *$ & 0,61 & $0,00 * * *$ \\
\hline \multicolumn{7}{|l|}{ Caractéristiques de l'enfant } \\
\hline Âge & 0,17 & 0,10 & 0,20 & $-0,02$ & 0,06 & $-0,01$ \\
\hline$\hat{A_{g e}}{ }^{2}$ & $-0,01 *$ & 0,01 & $-0,01 *$ & 0,00 & 0,00 & 0,00 \\
\hline Enfant du chef de ménage & $0,34 * * *$ & 0,20 & $0,01 * * *$ & $-0,12 * *$ & 0,11 & $-0,01 * *$ \\
\hline Sexe (masculin) & $-0,62 * * *$ & 0,20 & $0,02 * * *$ & $-0,24$ & 0,06 & $-0,01$ \\
\hline \multicolumn{7}{|l|}{ Caractéristiques du chef de ménage } \\
\hline Sexe (masculin) & $-0,24 * * *$ & 0,14 & $-0,04 * * *$ & $0,77^{*}$ & 0,45 & $0,04 *$ \\
\hline \multicolumn{7}{|l|}{ Niveau d'instruction (1) } \\
\hline Primaire & 0,02 & 0,11 & 0,02 & $-0,23 * * *$ & 0,22 & $-0,04 * * *$ \\
\hline Secondaire 1 , collège & $0,14 *$ & 0,19 & $0,02 *$ & $-0,07 * *$ & 0,34 & $-0,03 * *$ \\
\hline Secondaire 2, lycée & $0,63 * * *$ & 0,18 & $0,02 * * *$ & $-0,13 * * *$ & 0,66 & $-0,09 * * *$ \\
\hline Supérieur & $1,60 * * *$ & 0,22 & $0,01 * * *$ & $-0,23 * *$ & 0,20 & $-0,09 * *$ \\
\hline \multicolumn{7}{|l|}{ Statut sur le marché du travail } \\
\hline Secteur formel & $1,14 * * *$ & 0,38 & $0,04 * * *$ & $-0,37 *$ & 0,62 & $-0,01 *$ \\
\hline Secteur agricole & $-0,77 * * *$ & 0,06 & $-0,01 * * *$ & $0,28 *$ & 0,34 & $0,01 *$ \\
\hline Chômage & $-0,62$ & 0,13 & $-0,02$ & $0,12 * *$ & 0,11 & $0,05 * *$ \\
\hline \multicolumn{7}{|l|}{ Caractéristiques du ménage } \\
\hline Taille du ménage & $-0,05 * * *$ & 0,01 & $-0,01 * * *$ & 0,02 & 0,04 & 0,01 \\
\hline Nombre d'enfants de 0 à 4 ans & $-0,13 * *$ & 0,01 & $-0,05 * *$ & $0,02 * *$ & 0,01 & $0,02 * *$ \\
\hline Nombre d'enfants de 5 à 14 ans & $-0,07 * *$ & 0,10 & $-0,02 * *$ & $0,16^{* * *}$ & 0,40 & $0,02 * * *$ \\
\hline $\begin{array}{l}\text { Nombre d'individus de } 15 \text { à } \\
64 \text { ans }\end{array}$ & $0,40 * * *$ & 0,09 & $0,04 * * *$ & $0,07 * *$ & 0,10 & $0,01 * *$ \\
\hline Nombre d'individus $>64$ ans & $-0,10 * * *$ & 0,09 & $-0,10 * * *$ & $0,09 * * *$ & 0,02 & $0,06 * * *$ \\
\hline \multicolumn{7}{|l|}{ Niveau de vie du ménage } \\
\hline Revenu instrumenté & $0,02 * * *$ & 0,01 & $0,01 * * *$ & $-0,03 *$ & 0,02 & $-0,02 *$ \\
\hline \multicolumn{7}{|l|}{ Actifs } \\
\hline Possession de terres & $-0,01 * * *$ & 0,01 & $-0,01 * * *$ & $0,01 *$ & 0,01 & $0,01 *$ \\
\hline Maison inoccupée & $0,30 * * *$ & 0,12 & $0,13 * * *$ & $-0,19$ & 0,29 & $-0,01$ \\
\hline Accès au crédit & $0,23 * * *$ & 0,08 & $0,03 * * *$ & $-0,08^{*}$ & 0,18 & $-0,01 *$ \\
\hline \multicolumn{7}{|l|}{ Zones agro-écologiques (2) } \\
\hline Yaoundé & $0,63 * * *$ & 0,24 & $0,17 * * *$ & 0,37 & 0,70 & 0,01 \\
\hline Douala & $0,54 * *$ & 0,27 & $0,21 * *$ & 0,70 & 0,54 & 0,03 \\
\hline Autres villes & $0,39 * *$ & 0,18 & $0,14 * *$ & $-0,18^{*}$ & 0,13 & $-0,03 *$ \\
\hline Forêt & $0,24 *$ & 0,13 & $0,09 *$ & $-0,72 * * *$ & 0,17 & $-0,05 * * *$ \\
\hline Hauts Plateaux & 0,25 & 0,20 & 0,14 & $0,22 * *$ & 0,11 & $0,02 * *$ \\
\hline \multicolumn{7}{|l|}{ Chocs négatifs } \\
\hline Diminution du revenu & $-0,01 *$ & 0,08 & $-0,04 *$ & $0,17 * *$ & 0,12 & $0,06^{* *}$ \\
\hline Décès d'un parent & $-0,14 * *$ & 0,06 & $-0,01 * *$ & $0,10^{*}$ & 0,12 & $0,01 * *$ \\
\hline Distance école (km) & $-0,02 *$ & 0,01 & $-0,01^{*}$ & $0,05 * * *$ & 0,02 & $0,01^{* * *}$ \\
\hline Résidu & $-0,00 * *$ & 0,01 & $-0,00 * *$ & 0,01 & 0,00 & 0,00 \\
\hline$\rho$ (Wald; sig) & \multicolumn{6}{|c|}{$-0,24 * * *(3743,09 ; 0,00)$} \\
\hline Logarithme de vraisemblance & \multicolumn{6}{|c|}{$-3790,16$} \\
\hline$N$ & \multicolumn{6}{|c|}{13716} \\
\hline
\end{tabular}

Notes :*** significatif à $1 \% ; * *$ significatif à $5 \% ; *$ significatif à $10 \%$.

(1) Modalité de référence : sans instruction; (2) Modalité de référence : Savane.

Source : À partir de la base de données d'Ecam III, calcul des auteurs.

Premièrement, le revenu est effectivement endogène. Les résidus de l'équation d'instrumentation sont significatifs quant à la scolarisation des enfants. Par ailleurs, il ressort que le niveau de vie influence positivement les chances de scolarisation et négativement la probabilité de travailler. Les coefficients et les effets marginaux sont significatifs. Ainsi, dans l'ensemble, quand le revenu augmente d'une unité, la probabilité d'être scolarisé augmente de 1 point de pourcentage et la probabilité de travailler diminue de 2 points de pourcentage. Ces effets marginaux sont néanmoins faibles comparativement à ceux du revenu non instrumenté( ${ }^{(30)}$, ce qui signifie que l'impact du revenu sur le travail et la scolarisation des enfants tend à être surestimé lorsqu'il n'est pas instrumenté. Par ailleurs, le tableau A2 en annexe montre que les effets marginaux du revenu sur le travail des enfants sont beaucoup plus élevés en milieu rural. Autrement dit, l'impact de la pauvreté sur le travail des enfants est plus important dans

(30) Voir tableau A1 en annexes. 
les campagnes que dans les villes. Un tel résultat semble corroborer les conclusions de plusieurs études, même si certaines d'entre elles ont émis des réserves sur la validité de la relation entre les privations monétaires et le travail des enfants ${ }^{(31)}$. Il se pourrait que les ménages - surtout des zones rurales - utilisent le travail des enfants comme un substitut aux marchés des capitaux imparfaits. Étant donné qu'il leur est difficile de faire face aux variations de leur niveau de ressources en empruntant sur le marché, ils effectuent des transferts intergénérationnels et mettent au travail leurs enfants. On observe d'ailleurs dans le tableau A2 que le travail des enfants diminue lorsque les ménages ont accès au crédit, qu'ils soient installés en milieu rural ou en milieu urbain : il fait donc partie des stratégies de survie mises en œuvre par les ménages. En revanche, au fur et à mesure que le niveau de vie augmente, le revenu qu'apporte le travail des enfants n'est plus capital pour la survie du ménage qui cherche plutôt à garantir son bien-être futur en les scolarisant de plus en plus.

Deuxièmement, quel que soit l'échantillon considéré, la valeur du coefficient de corrélation $\rho$ entre la scolarisation et le travail des enfants est négative et significative: il existe donc une relation inverse entre les deux variables. Autrement dit, les facteurs qui accroissent la probabilité qu'ont les enfants d'aller à l'école diminuent celle qu'ils ont de travailler. La valeur absolue de $\rho$ est par ailleurs plus élevée en milieu rural qu'en milieu urbain.

Troisièmement, comme il fallait s'y attendre, les chocs monétaires et non monétaires constituent des déterminants significatifs du travail des enfants. Les estimations indiquent que lorsque les ménages sont victimes d'une diminution brutale de leur revenu, la probabilité de faire travailler les enfants augmente de 6 points de pourcentage. De même, lorsque les enfants perdent au moins un de leurs parents, leur probabilité de travailler croît de 1 point de pourcentage. Lorsqu'on compare les résultats des zones de résidence, il ressort que l'effet marginal de la diminution du revenu est plus élevé en zone rurale qu'en zone urbaine (4 points de pourcentage contre 2 ), ce qui signifie que les ménages ruraux sont plus sensibles aux chocs monétaires que les ménages urbains. Un tel résultat n'est pas étonnant dans la mesure où les ménages ruraux peuvent à tout moment subir un choc agricole ${ }^{(32)}$ qui mettrait en péril leur production et/ou leur autoconsommation. BEEgLE et ses coauteurs (2006) ont déjà avancé une telle conclusion, lors de leur étude sur les déterminants du travail des enfants en Tanzanie.

Quatrièmement, nous avons introduit dans le modèle deux variables susceptibles d'expliquer

(31) Cf. Coulombe (1998).

(32) Il peut s'agir de catastrophes naturelles (sécheresse, invasion d'insectes, etc.). également le travail et la scolarisation des enfants : la possession de terres et/ou d'une habitation inoccupée, qui sont des éléments constitutifs du patrimoine du ménage. La première variable est un facteur incitatif du travail des enfants, alors qu'elle diminue leur scolarisation. La seconde variable en revanche accroît significativement la probabilité d'être scolarisé mais n'est pas déterminante en ce qui concerne le travail. Lorsqu'on tient compte des zones de résidence, il ressort que la possession des terres par les ménages accroît le travail des enfants aussi bien dans les villes que dans les campagnes. Toutefois, l'impact est beaucoup plus important lorsque le ménage est établi en milieu rural. Ce résultat confirme celui obtenu par BHALOTRA et Heady (2003) au Pakistan et au Ghana. Ainsi, dans la plupart des pays en développement, les enfants constituent le plus souvent une main-d'œuvre indispensable pour les ménages, surtout en milieu rural.

Cinquièmement, des facteurs relatifs aux enfants eux-mêmes, aux chefs de ménage et à l'environnement dans lequel ils vivent expliquent également le travail et la scolarisation des enfants. Les résultats montrent ainsi que la fréquentation scolaire et le travail des enfants croissent avec l'âge, ce qui signifie qu'au fur et à mesure qu'ils grandissent, les enfants ont tendance à effectuer les deux activités en même temps. Mais, le signe négatif de la forme quadratique de cette variable pointe que l'impact de l'âge des enfants sur leur scolarisation prend la forme d'un U renversé, qui s'expliquerait par les départs précoces du système scolaire. Conformément aux résultats d'autres études(33), la position de l'enfant au sein du ménage a une influence sur sa mise au travail et sa scolarisation: les enfants du chef de ménage ont plus de chances de fréquenter une école $(+1$ point de pourcentage) et une probabilité moins grande de travailler $(-1$ point de pourcentage) que les autres enfants de la famille. On observe sensiblement la même tendance lorsqu'on tient compte du lieu de résidence des ménages ( $c f$. tableau A2). Les estimations du probit bivarié indiquent par ailleurs que les jeunes filles ont une probabilité plus grande de travailler et malheureusement moins de chances d'aller à l'école que les jeunes garçons. Toutefois, en zone urbaine, même si les jeunes filles fréquentent moins l'école que les garçons, elles ne sont pas particulièrement plus actives que ces derniers. Comme l'article de Cynthia B. Lloyd et Ann K. Blanc (1996), nos estimations montrent aussi que les enfants sont davantage scolarisés et travaillent moins dans les ménages gérés par une femme. Enfin, plus le chef de ménage est instruit, plus les enfants sont dispensés de travailler et plus leur scolarisation augmente. D'après les estimations, les effets marginaux de tous les niveaux d'éducation du chef de ménage sont

(33) Voir, par exemple, Grootaert (1998). 
significativement positifs pour la scolarisation des enfants et significativement négatifs pour le travail de ces derniers. Des parents bien éduqués ont la possibilité d'investir dans le capital humain de leurs enfants et de leur offrir un environnement propice à la scolarisation. Un meilleur niveau d'éducation est aussi associé à un meilleur accès aux marchés du crédit. Le fait que le chef de ménage ait un bon niveau d'instruction tend certainement à réorienter l'activité économique des membres du ménage vers des secteurs employant moins d'enfants. Ainsi, l'organisation de l'économie familiale tend-elle à mobiliser les enfants lorsque le chef de ménage est sans emploi ou se trouve à la tête d'une exploitation agricole ou encore travaille dans le secteur informel.

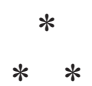

Cet article contribue à mettre en évidence des facteurs explicatifs du travail des enfants dans un pays d'Afrique, au sud du Sahara, le Cameroun. L'objectif était de montrer que, contrairement au résultat établi par BASU et VAN (1998), la pauvreté monétaire des ménages n'est pas le facteur prépondérant de la mise au travail des enfants. En effet, l'«axiome de luxe» de la pauvreté, hypothèse centrale mise en avant par ces deux auteurs, pose que la participation des enfants au travail ne prévaut que si le ménage a un niveau de vie inférieur à un certain seuil de subsistance. Comme nous l'avons relevé, selon d'autres hypothèses, la pauvreté n'est pas le seul facteur explicatif du travail des enfants : les imperfections du marché du crédit, la possession de terres et les chocs négatifs influençant le revenu des ménages peuvent être des déterminants plus significatifs. Notre étude se situe dans ce dernier cadre.

Sur le plan empirique, nous estimons un modèle probit bivarié qui suppose que le travail et la scolarisation des enfants sont des choix interdépendants, et mobilisons les données de la troisième Enquête camerounaise auprès des ménages (Ecam III). Nos résultats confirment le caractère multidimensionnel du phénomène du travail des enfants. Le niveau de vie des ménages mesuré par le revenu a ainsi un effet négatif sur le travail des enfants et positif sur leur scolarisation. Ces effets sont néanmoins faibles lorsque le revenu est instrumenté. Par ailleurs, plus le niveau de vie des ménages est élevé, plus la fréquentation scolaire augmente et la mise au travail des enfants diminue, ce qui signifie que les facteurs qui accroissent la probabilité qu'ont les enfants d'aller à l'école diminuent celle de travailler.

D'autres facteurs explicatifs du travail des enfants sont d'ampleur plus élevée. Les variables de choc indiquent que la baisse brutale du revenu est positivement et fortement associée à une augmentation du travail des enfants, tandis que la possession de terres nécessitant, à des fins d'exploitation, de la main-d'œuvre tend aussi à l'accroître. Ce dernier résultat traduit «le paradoxe de richesse»: dans une certaine mesure, les ménages qui possèdent des terres préfèrent utiliser leurs enfants dans leurs exploitations agricoles plutôt que de recourir à une main-d'œuvre externe. Enfin, le travail des enfants est aussi lié aux variables sociodémographiques, telles que la composition du ménage, le niveau d'éducation de son chef, le sexe, l'âge et le lien de parenté de l'enfant avec le chef de ménage.

De cette analyse, il ressort que la politique de réduction du travail des enfants devrait viser l'amélioration de la capacité des ménages à faire face aux chocs négatifs tels que des baisses brutales de revenus, par exemple par le développement de banques ou d'assurances publiques destinées aux ménages à bas revenu. On pourrait aussi envisager des modalités particulières de scolarisation pour les ménages agricoles. Enfin, parce qu'ils sont susceptibles d'être plus efficaces que des politiques générales de réduction de la pauvreté des ménages, la mise en place de "transferts conditionnels en espèces » pourrait être développée : ces programmes conditionnent le paiement d'aides sociales au fait que le bénéficiaire s'acquitte de certaines obligations, comme la nécessité de faire faire des visites médicales régulières et de vacciner ses enfants ou, pour ce qui nous concerne, de les envoyer à l'école en évitant de les faire travailler. Quoi qu'il en soit, afin de préciser encore les moyens de lutte possible contre le travail des enfants, il serait souhaitable d'étendre l'analyse en adoptant une approche dynamique : étudier les stratégies de gestion des risques des ménages selon cette perspective permettrait d'améliorer encore la compréhension des facteurs de la mise au travail des enfants. 


\section{Bibliographie}

Anker R. (2000), "L'économie du travail des enfants : un cadre d'analyse», Revue internationale $d u$ travail, vol. 139, n 3, pp. 289-317.

Baland J.-M., Robinson J. A. (2000), « Is child labor inefficient? », Journal of political economy, vol. 108, $\mathrm{n}^{\circ} 4$, pp. 663-679.

Bandara A., Dehejia R., Lavie-Rouse S. (2015), « The impact of income and non-income shocks on child labor: evidence from a panel survey of Tanzania », World development, vol. 67, pp. 218-237.

BASU K., VAN P. H. (1998), « The economics of child labor ", American economic review, vol. 88, $\mathrm{n}^{\circ} 3$, pp. 412-427.

Basu K., Tzannatos Z. (2003), « The global child labor problem: what do we know and what can we do?», World Bank economic review, vol. 17, $\mathrm{n}^{\circ}$ 2, pp. 147-173.

Beegle K., Deheja R. H., Gatti R. (2006), «Child labor and agricultural shocks", Journal of development economics, vol. 81, $\mathrm{n}^{\circ} 1$, pp. 80-96.

Bhalotra S., Heady C. (2003), « Child farm labor: the wealth paradox », World Bank economic review, vol. 17, $\mathrm{n}^{\circ} 2$, pp. 197-227.

BurEAu INTERNATIONAL DU TRAVAIL (BIT) (2002), Un avenir sans travail des enfants, Conférence internationale du travail, 90e session 2002, Rapport I(B), Genève, BIT.

Blunch N.-H., Verner D. (2000), « Revisiting the link between poverty and child labour: the Ghanaian experience ", Policy research working paper, $\mathrm{n}^{\circ} 2488$, Washington D. C., World Bank.

Bonnet M. (1993), «Le travail des enfants en Afrique», Revue internationale du travail, vol. 132, $\mathrm{n}^{\circ} 3$, pp. 411-430.

Boutin D. (2012), « Family farming, child labour and the wealth paradox: evidence from Mali », Economics bulletin, vol. 32, n 4, pp. 3471-3479.

Canagarajah S., Coulombe H. (1999), « Child labor and schooling in Ghana », Policy research working paper, $\mathrm{n}^{\circ}$ 1844, Washington D. C., World Bank.

Coudouel A., Hentschel J., Wodon Q. (2002), «Mesure et analyse de la pauvreté», MPRA Paper, $\mathrm{n}^{\circ}$ 10490, (World Bank).

Coulombe H. (1998), « Child labor and education in Côte d'Ivoire », unpublished paper written for the World Bank.

Couralet P.-E. (2003), «Le travail des enfants en Amérique latine», Documents de travail de l'OCDE: questions sociales, emplois et migrations, $\mathrm{n}^{\circ} 3$.

Denejia R., Gatti R. (2002), « Child labor: the role of income variability and access to credit across countries », NBER working paper, $\mathrm{n}^{\circ} 9018$.

Diallo Y., Étienne A., Mehran F. (2013), Tendances mondiales du travail des enfants de 2008 à 2012, Genève, BIT.
Dumas C. (2004), «Impact de la structure familiale sur les décisions parentales de mise au travail des enfants : le cas du Brésil, Revue d'économie du développement, vol. 12, $\mathrm{n}^{\circ} 1$, pp. 71-99.

Dumas C. (2007), « Why do parents make their children work? A test of the poverty hypothesis in rural areas of Burkina Faso », Oxford economic papers, vol. 59, $\mathrm{n}^{\circ} 2$, pp. 301-329.

Dumas C. (2013), «Market imperfections and child labor», World development, vol. 42, pp. 127-142.

Dumas C., Lambert S. (2008), Le travail des enfants : quelles politiques pour quels résultats ?, Paris, Éd. rue d'Ulm-ENS

Edmonds E. V. (2005), « Does child labor decline with improving economic status? ", Journal of human resources, vol. 40, $\mathrm{n}^{\circ} 1$, pp. 77-99.

EDMONDs E. V. (2006), « Child labor and schooling responses to anticipated income in South Africa », Journal of development economics, vol. 81, $\mathrm{n}^{\circ} 2$, pp. 386-414.

Grootaert C., Patrinos Harry H. (eds.) (1998), « The policy analysis of child labour: a comparative study », mimeo, Washington D. C., World Bank.

Guarcello L., Mealli F., Rosati F. C. (2010), " Household vulnerability and child labor: the effect of shocks, credit rationing, and insurance ", Journal of population economics, vol. 23, n 1, pp. 169-198.

INS (2008), Conditions de vie des populations et profil de pauvreté au Cameroun en 2007, Rapport principal de l'Ecam III, Yaoundé.

Janvry de A., Finan F., Sadoulet E., Vakis R. (2006), «Can conditional cash transfer programs serve as safety nets in keeping children at school and from working when exposed to shocks? ", Journal of development economics, vol. 79, $\mathrm{n}^{\circ} 2$, pp. 349-373.

Jensen P., Nielsen H. S. (1997), « Child labour or school attendance? Evidence from Zambia », Journal of population economics, vol. 10, $\mathrm{n}^{\circ} 4$, pp. 407-424.

Kobiané J.-F. (2009), La non-scolarisation des enfants issus de populations marginalisées au Burkina Faso: ampleur, causes et initiatives des pouvoirs publics, Rapport mondial de suivi sur l'éducation pour tous 2010, Paris, Unesco.

LAchaud J.-P. (2008), «Le travail des enfants et la pauvreté en Afrique : un réexamen appliqué au Burkina Faso », Économie et prévision, ${ }^{\circ} 186$, pp. 47-65.

Lloyd C. B., BlanC A. K. (1996), « Children’s schooling in sub-Saharan Africa: the role of fathers, mothers and others ", Population and development review, vol. 22, $\mathrm{n}^{\circ} 2$, pp. 265-298.

Maitra P., RAY R. (2002), « The joint estimation of child participation in schooling and employment: comparative evidence from three continents ", Oxford development studies, vol. 30, $\mathrm{n}^{\circ}$ 1, pp. 41-62. 
Nielsen H. S. (1998), «Child labor and school attendance: two joint decisions ", Working paper, $\mathrm{n}^{\circ}$ 98-15, Centre for labour market and social research, Aarhus (Danemark).

Ravallion M., Wodon Q. (1999), « Does child labor displace schooling? Evidence on behavior responses to an enrolment subsidy », Policy research working papers, $\mathrm{n}^{\circ}$ 2116, Washington D. C., World Bank.

RAY R. (2000), « Child labor, child schooling and their interaction with adult labor: empirical evidence for Peru and Pakistan ", The World Bank economic review, vol. $14, \mathrm{n}^{\circ} 2$, pp. 347-367.
Rivers D., Vuong Q. H. (1988), « Limited information estimators and exogeneity tests for simultaneous probit models ", Journal of econometrics, vol. 39, $\mathrm{n}^{\circ} 3$, pp. 347-366.

Thomas A. (2000), Économétrie des variables qualitatives, Paris, Dunod.

UNDERSTANDING CHILDREN's WORK (UCW) (2012), Cameroun : comprendre le travail des enfants et l'emploi des jeunes, Rapport de pays, juin 2012, Rome, UCW. 


\section{Annexes}

Tableau A1 : Probit bivarié des déterminants du travail et de la scolarisation des enfants âgés de 5 à 14 ans, relatifs au revenu non instrumenté

\begin{tabular}{|c|c|c|c|c|c|c|}
\hline \multirow{2}{*}{ Variables } & \multicolumn{3}{|c|}{ Scolarisation } & \multicolumn{3}{|c|}{ Travail des enfants } \\
\hline & Coefficient & Écart-type & Effet marginal & Coefficient & Écart-type & Effet marginal \\
\hline Constante & $-3,44 * * *$ & 0,38 & $0,00 * * *$ & $2,11 * * *$ & 0,33 & $0,00^{* * * *}$ \\
\hline \multicolumn{7}{|l|}{ Caractéristiques de l'enfant } \\
\hline Âge & $0,60 * * *$ & 0,06 & $0,01 * * *$ & $-0,04$ & 0,04 & $-0,01$ \\
\hline$\hat{A}^{2} e^{2}$ & $-0,03 * * *$ & 0,01 & $-0,00 * * *$ & 0,00 & 0,01 & 0,02 \\
\hline Enfant du chef de ménage & $0,43 * * *$ & 0,05 & $0,08 * * *$ & $-0,05^{*}$ & 0,08 & $-0,07 *$ \\
\hline Sexe (masculin) & $0,21 * * *$ & 0,04 & $0,02 * * *$ & $-0,01$ & 0,03 & $-0,01$ \\
\hline \multicolumn{7}{|c|}{ Caractéristiques du chef de ménage } \\
\hline Sexe (masculin) & $-0,16$ & 0,27 & $-0,01$ & $0,57^{*}$ & 0,43 & $0,03 *$ \\
\hline \multicolumn{7}{|l|}{ Niveau d'instruction (1) } \\
\hline Primaire & $0,51 * * *$ & 0,06 & $0,01 * * *$ & $-0,31 * * *$ & 0,09 & $-0,03 * * *$ \\
\hline Secondaire 1 , collège & $0,88^{* * *}$ & 0,09 & $0,02 * * *$ & $-0,26^{*}$ & 0,12 & $-0,02 *$ \\
\hline Secondaire 2, lycée & $0,64 * * *$ & 0,13 & $0,02 * * *$ & $-0,53 * * *$ & 0,14 & $-0,09 * * *$ \\
\hline Supérieur & $0,33^{*}$ & 0,21 & $0,02 *$ & $-0,54 * *$ & 0,23 & $-0,13 * *$ \\
\hline \multicolumn{7}{|c|}{ Statut sur le marché du travail (2) } \\
\hline Secteur formel & $0,35 * * *$ & 0,07 & $0,03 * * *$ & $-0,42 * * *$ & 0,05 & $-0,05 * * *$ \\
\hline Secteur agricole & $-0,37 * * *$ & 0,07 & $-0,04 * * *$ & $0,54 * * *$ & 0,06 & $0,23 * * *$ \\
\hline Chômage & $-0,06^{* *}$ & 0,08 & $-0,02 * *$ & $0,04 * *$ & 0,02 & $0,02 * *$ \\
\hline \multicolumn{7}{|l|}{ Caractéristiques du ménage } \\
\hline Taille du ménage & $-0,07 * * *$ & 0,01 & $0,01 * * *$ & 0,01 & 0,01 & 0,03 \\
\hline $\begin{array}{l}\text { Nombre d'enfants de } 0 \text { à } \\
4 \text { ans }\end{array}$ & $-0,15^{* *}$ & 0,06 & $-0,01 * * *$ & $0,02 * *$ & 0,90 & $0,03 * *$ \\
\hline $\begin{array}{l}\text { Nombre d'enfants de } 5 \text { à } \\
14 \text { ans }\end{array}$ & $-0,05^{* *}$ & 0,08 & $-0,05^{* *}$ & $0,13 * * *$ & 0,50 & $0,04 * * *$ \\
\hline $\begin{array}{l}\text { Nombre d'individus de } \\
15 \text { à } 64 \text { ans }\end{array}$ & $0,02 * * *$ & 0,03 & $0,04 * * *$ & $0,20 * *$ & 0,30 & $0,01 * *$ \\
\hline $\begin{array}{l}\text { Nombre d'individus > } \\
64 \text { ans }\end{array}$ & $-0,03 * * *$ & 0,06 & $-0,05 * * *$ & $0,14 * * *$ & 0,33 & $0,05 * * *$ \\
\hline \multicolumn{7}{|l|}{ Niveau de vie du ménage } \\
\hline Revenu (non instrumenté) & $0,15^{* * *}$ & 0,08 & $0,08 * * *$ & $-0,11 * * *$ & 0,10 & $-0,07 * * *$ \\
\hline \multicolumn{7}{|l|}{ Actifs } \\
\hline Possession de terres & $-0,01 * *$ & 0,01 & $-0,01 * *$ & $0,01^{*}$ & 0,01 & $0,07 *$ \\
\hline Maison inoccupée & $0,42 * * *$ & 0,10 & $0,07 * * *$ & 0,20 & 0,11 & 0,01 \\
\hline Accès au crédit & $0,25 * *$ & 0,08 & $0,02 * *$ & $-0,15^{*}$ & 0,02 & $0,08^{*}$ \\
\hline \multicolumn{7}{|c|}{ Zones agro-écologiques (Savane) } \\
\hline Yaoundé & $0,64 * * *$ & 0,24 & $0,03 * * *$ & 0,09 & 0,26 & $-0,61$ \\
\hline Douala & $0,90 * * *$ & 0,23 & $0,03 * * *$ & 0,33 & 0,31 & 0,12 \\
\hline Autres villes & $0,92 * * *$ & 0,11 & $0,03 * * *$ & $-0,19$ & 0,13 & $-0,04$ \\
\hline Forêt & $0,67 * * *$ & 0,09 & $0,01 * * *$ & $0,55 * * *$ & 0,14 & $0,08 * * *$ \\
\hline Hauts Plateaux & $1,13 * * *$ & 0,07 & $0,01 * * *$ & $0,17 * *$ & 0,10 & $0,04 * *$ \\
\hline \multicolumn{7}{|l|}{ Chocs négatifs } \\
\hline Diminution du revenu & $-0,16^{* *}$ & 0,10 & $-0,01 * *$ & $0,18 * *$ & 0,13 & $0,04 * *$ \\
\hline Décès d'un parent & $-0,03 * *$ & 0,08 & $-0,01 * *$ & $0,06^{*}$ & 0,10 & $0,03 *$ \\
\hline Distance école (km) & $-0,01$ & 0,01 & $-0,02$ & 0,02 & 0,01 & 0,01 \\
\hline$\rho$ (Wald; sig) & \multicolumn{6}{|c|}{$-0,05 * * *(797,51 ; 0,00)$} \\
\hline $\begin{array}{l}\text { Logarithme de } \\
\text { vraisemblance }\end{array}$ & \multicolumn{6}{|c|}{$-2069,62$} \\
\hline$N$ & & & & & & \\
\hline
\end{tabular}

Note : *** significatif à $1 \%$; ** significatif à $5 \%$; significatif à $10 \%$.

(1) Modalité de référence : sans instruction; (2) Modalité de référence : informel.

Source : À partir de la base de données d'Ecam III, calculs des auteurs. 
Tableau A2 : Probit bivarié des déterminants du travail et de la scolarisation des enfants âgés de 5 à 14 ans, relatifs au revenu instrumenté selon la zone de résidence

\begin{tabular}{|c|c|c|c|c|c|c|c|c|c|c|c|c|}
\hline \multirow{3}{*}{ Variables } & \multicolumn{6}{|c|}{ Urbain } & \multicolumn{6}{|c|}{ Rural } \\
\hline & \multicolumn{3}{|c|}{ Scolarisation } & \multicolumn{3}{|c|}{ Travail des enfants } & \multicolumn{3}{|c|}{ Scolarisation } & \multicolumn{3}{|c|}{ Travail des enfants } \\
\hline & $\begin{array}{l}\text { Coef- } \\
\text { ficient }\end{array}$ & $\begin{array}{l}\text { Écart- } \\
\text { type }\end{array}$ & $\begin{array}{c}\text { Effet } \\
\text { marginal }\end{array}$ & $\begin{array}{l}\text { Coef- } \\
\text { ficient }\end{array}$ & $\begin{array}{l}\text { Écart- } \\
\text { type }\end{array}$ & $\begin{array}{c}\text { Effet } \\
\text { marginal }\end{array}$ & $\begin{array}{l}\text { Coef- } \\
\text { ficient }\end{array}$ & $\begin{array}{l}\text { Écart- } \\
\text { type }\end{array}$ & $\begin{array}{c}\text { Effet } \\
\text { marginal }\end{array}$ & $\begin{array}{l}\text { Coeff- } \\
\text { icient }\end{array}$ & $\begin{array}{l}\text { Écart- } \\
\text { type }\end{array}$ & $\begin{array}{c}\text { Effet } \\
\text { marginal }\end{array}$ \\
\hline Constante & $-2,43 * * *$ & 0,37 & 0,00 & $2,64 * * *$ & 0,79 & 0,00 & $-2,59 * * *$ & 0,18 & 0,00 & $-2,21 * * *$ & 0,21 & 0,00 \\
\hline \multicolumn{13}{|c|}{ Caractéristiques de l'enfant } \\
\hline Âge & $0,21 * *$ & 0,10 & 0,09 & $-0,11$ & 0,08 & $-0,01$ & 0,04 & 0,03 & 0,02 & 0,01 & 0,02 & $-0,01$ \\
\hline$\hat{A g e}^{2}$ & $-0,01 * * *$ & 0,01 & $-0,01 * * *$ & 0,01 & 0,01 & 0,01 & $-0,01 * * *$ & 0,01 & $-0,01 * * *$ & $-0,01 *$ & 0,01 & 0,00 \\
\hline $\begin{array}{l}\text { Enfant du chef de } \\
\text { ménage }\end{array}$ & $0,57 * * *$ & 0,08 & $0,17^{* * *}$ & $-0,19 * *$ & 0,09 & $-0,02^{* *}$ & $0,21^{* * *}$ & 0,04 & $0,01^{* * *}$ & $-0,18^{* * *}$ & 0,04 & $-0,01 * * *$ \\
\hline Sexe (masculin) & $0,05 * * *$ & 0,04 & $0,02 * * *$ & 0,08 & 0,01 & 0,02 & $0,28^{* * *}$ & 0,02 & $0,01 * * *$ & $-0,06^{*}$ & 0,01 & $-0,03 *$ \\
\hline \multicolumn{13}{|c|}{ Caractéristiques du chef de ménage } \\
\hline Sexe (masculin) & $-0,11^{*}$ & 0,06 & $-0,02 *$ & $0,14 * *$ & 0,07 & $0,02 * *$ & $-0,03$ & 0,04 & $-0,01$ & $-0,01$ & 0,04 & $-0,01$ \\
\hline \multicolumn{13}{|c|}{ Niveau d'instruction (1) } \\
\hline Primaire & $0,39 *$ & 0,21 & $0,17 *$ & $-0,04$ & 0,10 & $-0,01$ & $0,17 * * *$ & 0,04 & $0,17 * * *$ & $-0,11 * * *$ & 0,04 & $-0,01 * * *$ \\
\hline Secondaire 1, collège & $0,54^{*}$ & 0,37 & $0,22 *$ & $-0,17$ & 0,16 & $-0,01$ & $0,52 * * *$ & 0,06 & $0,21 * * *$ & $-0,47 * * *$ & 0,06 & $-0,01 * * *$ \\
\hline Secondaire 2, lycée & $0,30 * *$ & 0,41 & $0,19 * *$ & $-0,01 *$ & 0,25 & $-0,04 *$ & $0,78^{* * *}$ & 0,11 & $0,21 * * *$ & $-0,69 * * *$ & 0,11 & $-0,01 * * *$ \\
\hline Supérieur & $0,40^{*}$ & 0,65 & $0,15^{*}$ & $-0,07 *$ & 0,52 & $-0,03^{*}$ & $1,48^{* * * *}$ & 0,27 & $0,15^{* * *}$ & $-1,49 * * *$ & 0,29 & $-0,04 * * *$ \\
\hline \multicolumn{13}{|c|}{ Statut sur le marché du travail (2) } \\
\hline Secteur formel & $1,01 * * *$ & 0,32 & $0,15^{* * *}$ & $-0,01$ & 0,48 & $-0,07$ & -- & -- & -- & -- & -- & -- \\
\hline Secteur agricole & $-0,84 * * *$ & 0,07 & $-0,01 * * *$ & $0,01 * * *$ & 0,13 & $0,05^{* * *}$ & $-0,18^{* *}$ & 0,07 & $-0,07 * *$ & $0,20 * * *$ & 0,07 & $0,02 * *$ \\
\hline Chômage & 0,01 & 0,13 & 0,05 & 0,04 & 0,04 & 0,01 & $-0,02$ & 0,13 & 0,02 & 0,12 & 0,08 & 0,04 \\
\hline \multicolumn{13}{|c|}{ Caractéristiques du ménage } \\
\hline Taille du ménage & $-0,05 * *$ & 0,02 & $-0,01^{* *}$ & $-0,01$ & 0,03 & $-0,01$ & $-0,07 * * *$ & 0,01 & $0,01 * * *$ & $0,06 * * *$ & 0,01 & 0,01 \\
\hline $\begin{array}{l}\text { Nombre d'enfants de } \\
0 \text { à } 4 \text { ans }\end{array}$ & $0,02 * *$ & 0,91 & $0,01 * *$ & $0,02 * *$ & 0,02 & $0,02 * *$ & $-0,02$ & 0,04 & $-0,01$ & 0,09 & 0,01 & 0,02 \\
\hline $\begin{array}{l}\text { Nombre d'enfants de } \\
5 \text { à } 14 \text { ans }\end{array}$ & $-0,03 * *$ & 0,16 & $-0,01 * *$ & 0,06 & 0,02 & 0,07 & $-0,42$ & 0,01 & $-0,00$ & 0,10 & 0,01 & 0,01 \\
\hline $\begin{array}{l}\text { Nombre d'individus } \\
\text { de } 14 \text { à } 64 \text { ans }\end{array}$ & $-0,03 * *$ & 0,12 & $-0,01 * *$ & $0,06^{*}$ & 0,08 & $0,08^{*}$ & $-0,10$ & 0,01 & $-0,03$ & $-0,03 *$ & 0,05 & $-0,01 *$ \\
\hline $\begin{array}{l}\text { Nombre d'individus } \\
>64 \text { ans }\end{array}$ & 0,05 & 0,21 & 0,01 & $-0,02$ & 0,02 & $-0,08$ & $-0,23$ & $-0,01$ & $-0,06$ & 0,01 & 0,02 & 0,02 \\
\hline \multicolumn{13}{|c|}{ Niveau de vie du ménage (3) } \\
\hline Revenu instrumenté & $0,01 * * *$ & 0,01 & $0,01 * * *$ & $-0,02 * *$ & 0,00 & $-0,01^{* *}$ & $0,02 * * *$ & 0,00 & $0,02 * * *$ & $-0,03 *$ & 0,01 & $-0,02 *$ \\
\hline \multicolumn{13}{|l|}{ Actifs } \\
\hline Possession de terres & $-0,01 *$ & 0,00 & $-0,00^{*}$ & 0,01 & 0,00 & 0,01 & $-0,02 * * *$ & 0,01 & $-0,01 * * *$ & $0,02 * * *$ & 0,01 & $0,02 * * *$ \\
\hline Maison inoccupée & 0,08 & 0,19 & 0,06 & $-0,02 *$ & 0,01 & $-0,01^{*}$ & $0,07^{* * *}$ & 0,06 & $0,05^{* * *}$ & $-0,22 * * *$ & 0,06 & $-0,01 * * *$ \\
\hline Accès au crédit & $0,11 *$ & 0,13 & $0,06^{*}$ & $-0,01 *$ & 0,01 & $-0,01^{*}$ & $0,57^{* * *}$ & 0,08 & $0,07 * * *$ & $-0,58 * * *$ & 0,08 & $-0,01 * * *$ \\
\hline \multicolumn{13}{|c|}{ Zones agro-écologiques (Yaoundé, Savane) } \\
\hline Douala & $-0,50 * *$ & 0,20 & $0,04 * *$ & $0,48 * *$ & 0,28 & 0,04 & -- & -- & -- & -- & -- & -- \\
\hline Autres villes & $0,33 * * *$ & 0,07 & $0,06^{* * *}$ & $-0,19 * *$ & 0,10 & $-0,02$ & -- & -- & -- & -- & -- & -- \\
\hline Forêt & -- & -- & -- & -- & -- & -- & $0,49 * * *$ & 0,08 & $0,13 * * *$ & $0,20 * * *$ & 0,06 & $0,02 * * *$ \\
\hline Hauts Plateaux & -- & -- & -- & -- & -- & -- & $1,04 * * *$ & 0,07 & $0,30 * * *$ & $-0,33 * * *$ & 0,04 & $-0,01 * * *$ \\
\hline \multicolumn{13}{|l|}{ Chocs négatifs } \\
\hline $\begin{array}{l}\text { Diminution de } \\
\text { revenu }\end{array}$ & $-0,02^{*}$ & 0,09 & $-0,01^{*}$ & $0,10^{*}$ & 0,13 & $0,02 *$ & $-0,02^{*}$ & 0,06 & $-0,07^{*}$ & $0,05^{*}$ & 0,06 & $0,04 *$ \\
\hline Décès d'un parent & $-0,17^{* *}$ & 0,08 & $-0,03 * *$ & $0,28^{*}$ & 0,11 & $0,01 *$ & $-0,20 * *$ & 0,05 & $-0,02 * *$ & $0,19^{* * *}$ & 0,06 & $0,01 * * *$ \\
\hline Distance école (km) & $-0,01$ & 0,01 & $-0,01$ & $0,04 *$ & 0,03 & $0,01 *$ & $-0,01$ & 0,01 & $-0,01$ & $0,03 *$ & 0,01 & $0,05^{*}$ \\
\hline Résidu & $-0,00^{*}$ & 0,00 & $0,00^{*}$ & $0,00^{*}$ & 0,01 & $0,00^{*}$ & 0,00 & 0,00 & 0,00 & 0,00 & 0,00 & 0,00 \\
\hline$\rho$ (Wald; sig) & \multicolumn{6}{|c|}{$-0,23^{* * *}(1074,14 ; 0,00)$} & \multicolumn{6}{|c|}{$-0,40 * * *(588,94 ; 0,00)$} \\
\hline $\begin{array}{l}\text { Logarithme de } \\
\text { vraisemblance }\end{array}$ & \multicolumn{6}{|c|}{$-3732,67$} & \multicolumn{6}{|c|}{$-4242,95$} \\
\hline$N$ & & & 85 & 94 & & & & & 51 & & & \\
\hline
\end{tabular}

Note : *** significatif à $1 \%$; ** significatif à $5 \%$; * significatif à $10 \%$.

(1) Modalité de référence : sans instruction; (2) Modalité de référence : informel; (3) Modalité de référence : très pauvre.

Source : À partir de la base de données d'Ecam III, calculs des auteurs. 